\title{
StoryLab: Laboratorio de historias audiovisuales a través del intercambio cultural
}

\section{StoryLab: Audiovisual Stories Laboratory through cultural exchange}

\author{
If Sandra Carolina Patin̄o Ospina* \\ Sergio Andrés Forero Machado**
}

C) Recepción: $12 / 09 / 2019$

(C) Aprobación: $23 / 10 / 2019$

(C) Publicación: 19/12/2019

Para citar este artículo:

入 Patiño Ospina, S. C., \& Forero Machado, S.A. (2019). StoryLab: Laboratorio de historias audiovisuales a través del intercambio cultural. Indagare, (7), 68-75. https://doi.org/10.35707/indagare/709

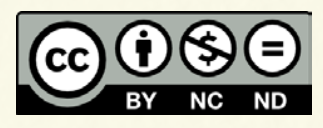

* Coinvestigadora del proyecto StoryLab Skills Training for Democratised. Universidad de Ibagué, ORCID: 0000-00016115-1978. Correo electrónico: sandra.patino@unibague.edu.co

** Grupo de Investigación Rastro Urbano, Universidad de Ibagué, ORCID: 0000-0002-6362-4078. Correo electrónico: sergio.forero@unibague.edu.co 


\title{
Resumen
}

"Laboratorio de historias para el desarrollo de habilidades en narraciones audiovisuales para la democratización de industrias fílmicas" (StoryLab Skills Training for Democratised Film Industries), es un proyecto de investigación global realizado en varios países en vías de desarrollo. En esta ocasión, Erik Knudsen, investigador principal y director del proyecto habla sobre el vínculo y las alianzas que se gestaron con la Universidad de Ibagué en el marco del proyecto adelantado en Ibagué, Colombia, y de la importancia de este para lograr un impacto real en comunidades marginadas a partir del entrenamiento en habilidades para la narración de sus propias historias y relatos.

\begin{abstract}
"StoryLab Skills Training for Democratised Film Industries" is a global research project carried out in several developing countries. On this occasion, Erik Knudsen, principal researcher and director of the project talks about the linkage and alliances that were formed with Universidad de Ibagué within the framework of the project developed in Ibagué, Colombia; and the importance of this to achieve a real impact on marginalized communities from training in storytelling skills of their own stories and narratives.
\end{abstract}

Palabras claves: StoryLab, narraciones audiovisuales, sectores democráticos, audiovisual, Ibagué.

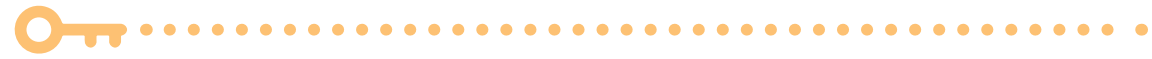

Key words: StoryLab, audiovisual stories, democratized communities, audiovisual, Ibagué.

\section{Introducción}

El proyecto "Laboratorio de historias para el desarrollo de habilidades en narraciones audiovisuales para la democratización de industrias fílmicas" (StoryLab Skills Training for Democratised Film Industries), que en su primera etapa se denominó: “Red Internacional de Investigación para el Desarrollo de laboratorios de narrativas audiovisuales" (StoryLab International Film Development Research Network) fue financiado, entre otros, por Arts and Humanities Research Council-UK (AHRC) en los últimos cuatro años. El equipo de investigadores en estas dos fases del proyecto lo han integrado: Nico Meissner de Multimedia University, Malasia y Griffith University, Australia; Sarah Kuntoh de National Film and Television Institute, Ghana; Carolina Patiño Ospina de la Universidad de Ibagué y el director general del proyecto Erik Knudsen de University of Central Lancashire, Reino Unido. 
El objetivo de este proyecto está centrado en indagar si la democratización de los medios de producción y distribución cinematográfica libera las formas narrativas de los realizadores en los países en desarrollo, de la hegemonía de las formas narrativas occidentales y en democratizar el acceso a la formación como aprendices y luego como mentores, por medio de talleres que faciliten la creación de historias y relatos audiovisuales que beneficien su progreso personal, creativo y el de su entorno. Dichos talleres se han realizado en las universidades participantes del proyecto en Ghana, Malasia y Colombia. La relevancia e importancia de adelantar este tipo de talleres, se sustenta en que estos tres países en vía de desarrollo comparten la característica de tener poblaciones que comúnmente son excluidas de acceder a este tipo de proyectos y laboratorios exploratorios que brindan espacios propicios, para crear historias individuales y colectivas que a la postre nutran sus creaciones en red para el fortalecimiento de sus lazos comunitarios en el plano artístico, cultural y, por supuesto, audiovisual.

En su última visita a Colombia, para la realización del StoryLab II-Ibagué que se llevó a cabo en la Universidad de Ibagué, como parte de la metodología de esta investigación ${ }^{1}$, en la primera semana de junio, el director de este proyecto, Erik Knudsen, respondió a varias inquietudes sobre el mismo, su vínculo con la Universidad y su experiencia como investigador en el contexto colombiano, entre otros aspectos. Él actualmente es profesor

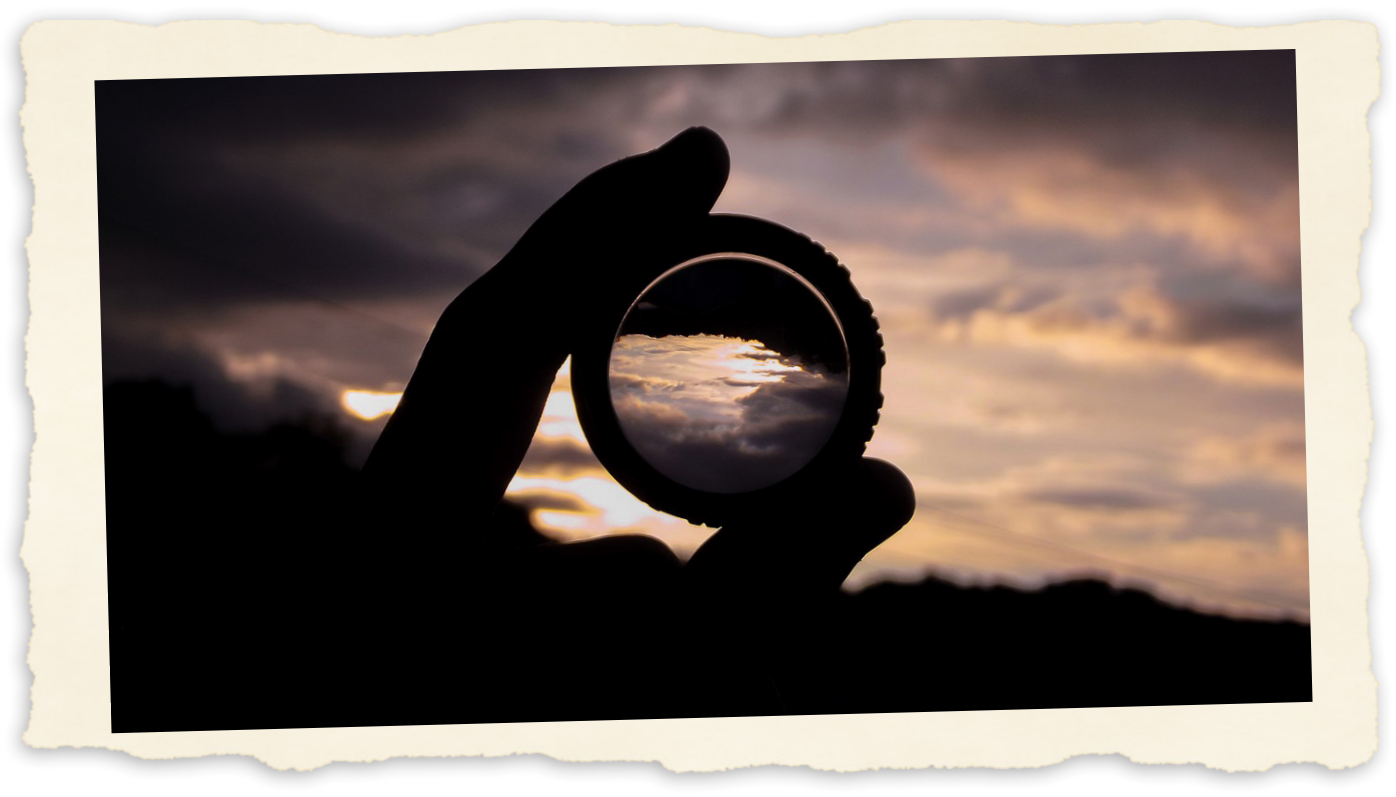

\footnotetext{
${ }^{1}$ La metodología de investigación de este proyecto parte de prácticas como la etnomusicología y la autoetnografía. En ese sentido y tomando como base dichos enfoques, se apela a una exploración metodológica que implica la participación activa y la inmersión de los investigadores en la cultura de los países a los que viajan como Colombia, Ghana y Kuala Lumpur, de manera que la lectura e interpretación de lo escuchado, observado y vivido en el marco del proceso de investigación, provenga de las actividades formales e informales que se realizan y comparten con los participantes, acorde a la agenda de cada taller de StoryLab.
} 
titular de la Escuela de Cine, Medios de Comunicación y Performance de University of Central Lancashire, Reino Unido. Es autor de los libros Creative documentary (2012) y Finding the personal voice in filmmaking (2018). También ha escrito y dirigido decenas de programas radiales y de televisión para la BBC, así como cortometrajes de ficción y documentales con distinciones en diferentes festivales de cine, como guionista, director y editor, con el sello de su productora de cine independiente: One Day Films en el Reino Unido. Estudió Producción Cinematográfica en la Universidad de York en Toronto, Canadá, donde se graduó con honores en una Licenciatura en Bellas Artes con énfasis en Producción Cinematográfica, en 1983. Fue profesor de Cultura Visual y Digital en la University of Bournemouth por varios años. En 2002 obtuvo su doctorado en la University of Salford. Hasta septiembre de 2007, fue director de la Maestría en Producción Documental y de la Maestría en Producción de Películas de Ficción en la Escuela de Música de Medios y Actuación de la Universidad de Salford. Dirige regularmente talleres como profesor invitado en escuelas internacionales de cine en diferentes continentes, como la Escuela Internacional de Cine y Televisión San Antonio de los Baños, Cuba, donde fue el jefe del Departamento de Edición entre 2001 y 2009.

Figura 1. Fotografía de Erik Knudsen de Erik, director general del proyecto StoryLab, durante el taller de StoryLab Research Network en la Universidad de Ibagué (Colombia)

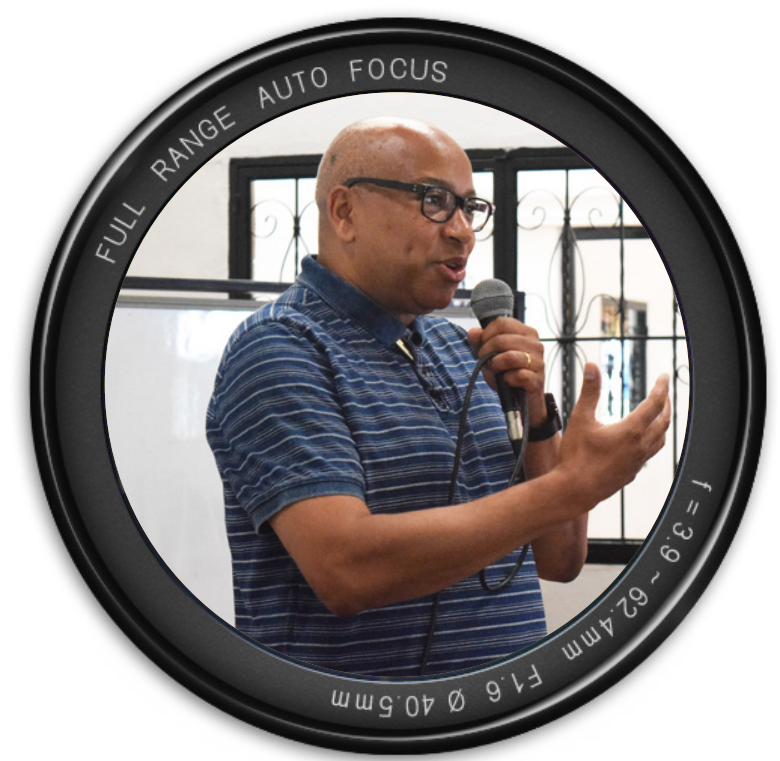

Fuente: Fotografía de Juan Pablo Ramírez. 
INDAGARE Ze-ISSN:2357-5042. Numero 7 (2019)- Universidad de Ibagué dor: https:/doirorg/0357077

¿Cómo fue su experiencia en la Universidad de Ibagué, al tener en cuenta que es la segunda vez que nos visita?

Personalmente encontré muy gratificante e inspirador visitar la Universidad de Ibagué y sé que mis colegas, el doctor Nico Meisner de Griffith Film School en Australia y Sarah Kuntoh de National Film and Television Institute (NAFTI) en Ghana, también piensan lo mismo sobre estas visitas a Ibagué.

Nuestro compromiso académico con la Universidad ha sido muy agradable y para nosotros es muy reconfortante ver cómo nuestra colega, la doctora Carolina Patiño, tiene un gran apoyo institucional y de sus estudiantes. Nos hemos sentido bien recibidos por toda la comunidad y valoramos muchísimo el hecho de que la Universidad de Ibagué aprecie y valore nuestra investigación. También pensamos que la ubicación de la Universidad y las instalaciones donde se han realizado nuestros talleres son muy convenientes y agradables para fomentar la creatividad. Nos hemos sentido como en casa $\mathrm{y}$ anhelamos visitarlos muy pronto.

En su opinión, ¿cuál es la importancia de establecer alianzas entre universidades de diferentes contextos para el desarrollo de proyectos de investigación?

Muchos de los retos a los que nos enfrentamos hoy, son desafíos compartidos por personas de todo el mundo en los que la colaboración, el intercambio de experiencias y las buenas prácticas hacen una gran diferencia. Naciones Unidas identificó diecisiete Objetivos de Desarrollo Sostenible que reflejan estos retos comunes y está claro que mediante la conformación de redes de investigación internacionales, podemos aprovechar al máximo los beneficios que estas ofrecen. StoryLab Skills Training for Democratised Film Industries es un proyecto único, construido desde enfoques innovadores con miras al desarrollo de historias para la realización de productos audiovisuales, y aprovecha estas oportunidades para que los realizadores y las comunidades compartan sus experiencias sobre temas e historias personales, locales y nacionales, a través del intercambio cultural.

Estos enfoques en el desarrollo de habilidades impulsados por talleristas, quienes previamente en el rol de aprendices fueron formados para convertirse en mentores en el marco del proyecto, pueden desempeñar un papel importante en el fomento de nuevas colaboraciones creativas, oportunidades comerciales y de formación profesional, no solo dentro de las comunidades de estos países, sino en el ámbito internacional. Esto permite que se escuchen más voces, a menudo marginadas, para que participen creando sus historias, desde su propia identidad, brindándoles así más oportunidades, desafíos y desarrollo global. 


\section{¿Cómo logró el vínculo con la Universidad de Ibagué?}

Tuve el privilegio de haber sido el supervisor de doctorado de la doctora Carolina Patiño, en University of Salford en el Reino Unido, cuando trabajaba allí como profesor. Mantuvimos nuestra relación de trabajo y en el desarrollo del proyecto StoryLab, ella fue una de las dos personas que identifiqué e invité como colaboradora e investigadora. La doctora Patiño goza de una experiencia y conocimientos particulares que han sido fundamentales para el éxito y buen avance del proyecto. Cuando ella se incorporó posteriormente a la Universidad de Ibagué, fue inevitable que estableciéramos una relación de trabajo con la Universidad. Yo no sigo a las instituciones, sigo a las buenas personas.

\section{¿Qué iniciativas han resultado del establecimiento de esta red internacional?}

"El Laboratorio de historias para el desarrollo de habilidades en narraciones audiovisuales para la democratización de industrias fílmicas" logra un impacto considerable en los participantes y está dando lugar a una serie de talleres que se adelantan en todo el mundo. No solo hemos impartido talleres en Ibagué, sino en Kuala Lumpur, Malasia, Accra, Ghana, California, Estados Unidos y en Zagoria, Grecia. Todas estas comunidades de ciudadanos realizadores y de cineastas independientes están ahora conectados por los resultados subyacentes de los procesos de investigación de nuestros proyectos, así como entre ellos en los diferentes talleres o StoryLab. Se han concretado nuevas colaboraciones, incluso internacionales. Se han creado nuevos proyectos e implementado nuevos enfoques para la enseñanza y el aprendizaje de la narración audiovisual. Además, uno de los Consejos de investigación más importantes en el Reino Unido, el Arts and Humanities Research Council, ha financiado dos etapas del proyecto StoryLab, como reconocimiento al impacto que este tiene en el ámbito mundial.

\section{¿Por qué el grupo de investigación eligió a Colombia como uno de los sitios para} desarrollar el proyecto?

Después de haber realizado varios talleres en la Fase 1 del proyecto StoryLab en Kuala Lumpur, Accra e Ibagué, estábamos agradecidos por el compromiso y dedicación de la doctora Carolina Patiño, la Universidad de Ibagué y los participantes colombianos, así como por la diversidad geográfica de la ciudad y la vasta vegetación del campus de la Universidad. Por ello, cuando tuvimos la oportunidad de profundizar nuestro trabajo de manera más específica en un país, no hubo duda al escoger a Colombia. Ibagué en particular fue la opción obvia. Nuestro primer taller en Ibagué tuvo un impacto significativo. Además, hemos encontrado que Colombia presenta un contexto sociopolítico interesante para esta investigación. 
Figura 2. Fotografía de los integrantes del proyecto StoryLab en Colombia

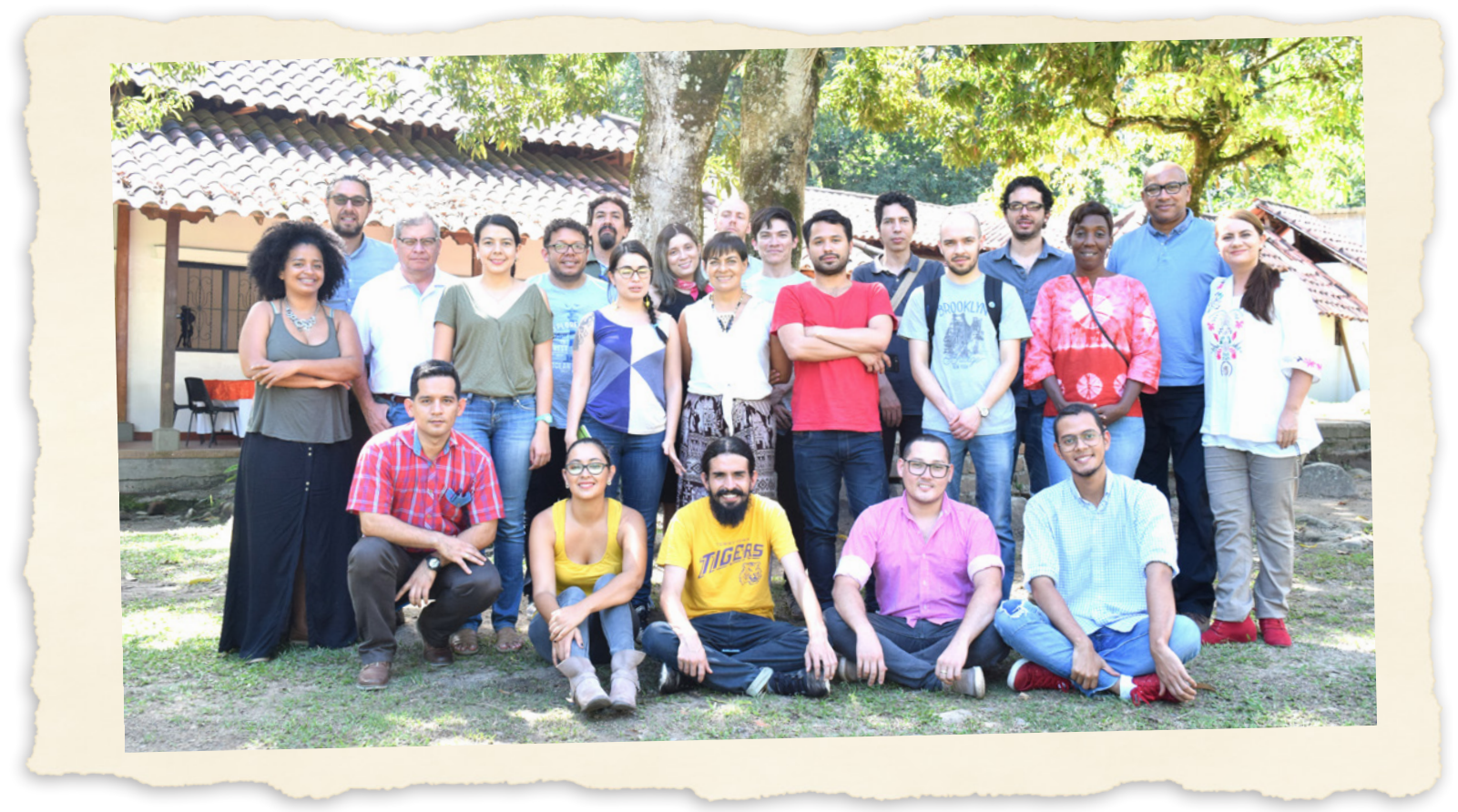

Fuente: Fotografía de Juan Pablo Ramírez.

\section{¿Cómo percibió la recepción de StoryLab en Colombia?}

Nos ha motivado e inspirado la respuesta positiva que hemos recibido de todos los participantes colombianos en nuestros talleres y el compromiso que ellos, el personal de la Universidad de Ibagué y la doctora Carolina Patiño y su equipo han mostrado en el proyecto.

\section{¿Qué impacto puede tener este proyecto de investigación en la región y el país?}

Todavía estamos en el proceso de evaluación del impacto del proyecto en Colombia. En primer lugar, StoryLab ha logrado efectos tanto positivos como significativos en los más de 20 participantes que han hecho parte de los talleres. Las experiencias de trabajo en sus propias comunidades han cambiado a medida que han adoptado aprendizajes y experiencias del proyecto StoryLab en sus propias situaciones y contextos. En segundo lugar, estamos difundiendo el componente entendido como "mentor-tallerista", que significa que un número de participantes irá a dos regiones diferentes de Colombia (Santa 
Marta y Tolima) para trabajar con varios tipos de comunidades en el desarrollo de historias y habilidades narrativas para la realización de productos audiovisuales. Esto ayudaría a empoderar a un número significativo de personas (aproximadamente 30 a 40) para que adquieran habilidades avanzadas de narración audiovisual, las que posteriormente puedan aplicar para lograr un impacto en sus comunidades, en su entorno empresarial, personal y cultural.

El objetivo es que los resultados de la investigación se transmitan de acuerdo a los valores de la integridad, la autenticidad y la apertura, que son pilares del proyecto, como base fundamental en la enseñanza de esta innovadora formación en habilidades para narrar historias en formato audiovisual. Finalmente, los hallazgos desarrollados a partir del trabajo que se lleva a cabo en Colombia, serán compartidos y difundidos a los participantes de StoryLab en otros países - incluidos los países existentes de StoryLab de Malasia, Ghana, Estados Unidos y Grecia-, y así ampliar significativamente el impacto del trabajo que se realiza actualmente.

Para obtener más información acerca de este proyecto StoryLab Skills Training for Democratised Film Industries y sobre los diferentes laboratorios que se han realizado y se continuarán impartiendo como parte de la metodología, se puede acceder a la página web: storylabnetwork.com. Adicionalmente, se puede visitar el sitio web: onedayfilms. com, para reconocer el impacto de la investigación en las personas que han participado en el proyecto como investigadores, aprendices y mentores, entre otros.

\section{Referencias}

Knudsen, E., De Jong, W. \& Rothwell, J. (2012). Creative documentary: Theory and practice. London, England: Pearson.

Knudsen, E. (2018). Finding the personal voice in filmmaking. London, England: Palgrave Macmillan.

One Day Films. (s.f.). One Day Films. Producers \& Distributors of Quality, Thought-Provoking Independent Films. Recuperado de https://www.onedayfilms.com/

Storylab. (2018). StoryLab Colombia Success. Recuperado de https://www.storylabnetwork. com/2017/09/26/storylab-colombia-success/ 